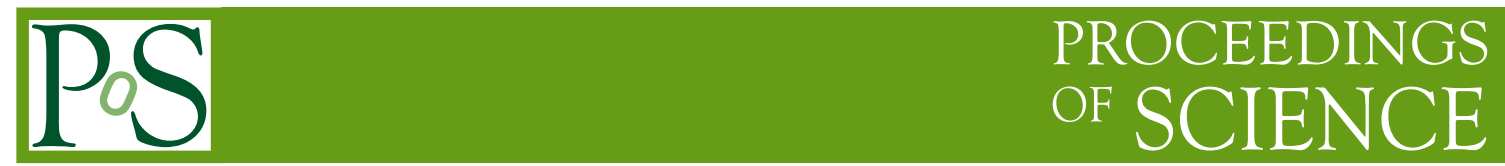

\title{
Recent results on heavy flavour in small and large systems from $\mathrm{LHCb}$
}

\author{
Benjamin Audurier for the $\mathrm{LHCb}_{\text {collaboration }}{ }^{a}$ \\ ${ }^{a}$ Laboratoire Leprince-Ringuet, \\ École polytechnique, 91128 Palaiseau Cedex - France \\ E-mail: audurier@llr.in2p3.fr
}

In these proceedings, we report on the latest results on heavy flavour production obtained by the LHCb collaboration in small and large systems. After a short introduction, we present results on open-beauty mesons measurements and their ratios in $p p$ collisions at $\sqrt{s}=7,8$ and $13 \mathrm{TeV}$. Then, we focus on the first measurement of prompt double charm production in $p \mathrm{~Pb}$ collisions at $\sqrt{s_{\mathrm{NN}}}$ $=8.16 \mathrm{TeV}$ and the studies of double parton scattering.

The Eighth Annual Conference on Large Hadron Collider Physics-LHCP2020

25-30 May, 2020

online 


\section{Introduction}

Initially designed to study the matter-antimatter asymmetry with b-hadrons and rare decays, $\mathrm{LHCb}[1]$ is a one-arm spectrometer fully instrumented at forward rapidity. Over the years, the detector has shown great performances [2] for particle identification capabilities, vertex reconstruction, mass and momentum resolution down to zero $p_{\mathrm{T}}$. Since 2013 and the recording of the first $p \mathrm{~Pb}$ data sample, $\mathrm{LHCb}$ has turned into a multipurpose detector with an ever expanding heavy-ion physics program, thanks to its unique capability among the LHC experiments to run both in collider and fixed-target mode via the injection of noble gases at the interaction point.

The versatility of the detector allows the collaboration to study heavy-flavour (HF) hadrons over a large range of colliding energy and colliding species. While many recent results in $p p$ collisions at $\sqrt{s}=13 \mathrm{TeV}$ have been obtained in the sector of hadron spectroscopy [3, 4], we first turn our attention to b-hadron production fractions and the associated measurement of fragmentation fraction ratios. Then, we focus on $p \mathrm{~Pb}$ collisions at $\sqrt{s_{\mathrm{NN}}}=8.16 \mathrm{TeV}$. Here also, many results have been obtained in the beauty sector [5], but only the recent double charm production results are discussed in these proceedings.

\section{Recent results on $p p$ collisions: b-hadron ratios}
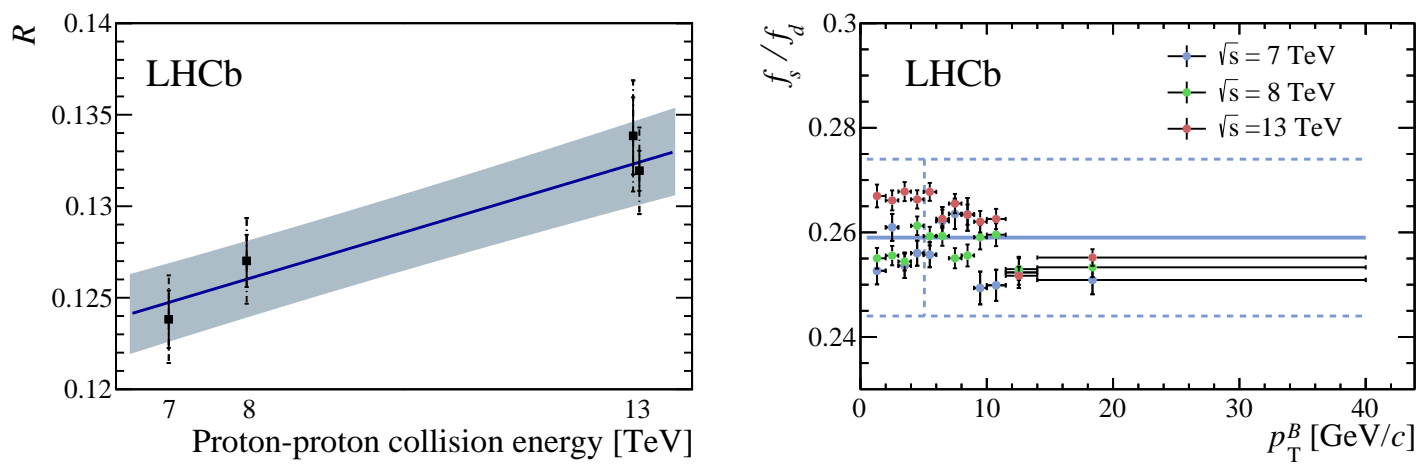

Figure 1: Left: efficiency-corrected $B_{s}^{0} \rightarrow J / \psi \phi$ to $B^{+} \rightarrow J / \psi K^{+}$yield ratios $(R)$ at different $p p$ collision energies with the total (uncorrelated, including statistical) uncertainties denoted by dashed (solid) error bars. A linear fit is shown with the blue solid line, the blue band denotes the $68 \%$ confidence region. Right: $R$ in bins of mesons $p_{\mathrm{T}}^{B}$ in the three collision energies. The ratios are scaled to match the measured $f_{s} / f_{d}$ value (horizontal blue lines, the $\pm 1 \sigma$ interval is indicated by the dashed blue lines) at the positions indicated by the vertical gray lines.

Heavy quarks (i.e charm and beauty) are natural probes to test $\mathrm{pQCD}$ factorization thanks to their relatively high mass compared to $\Lambda_{Q C D}$. By measuring heavy quarks (HQs) production in high-energy $p p$ collisions, one can test the universality of the non perturbative parts of $\mathrm{pQCD}$ models such as parton distribution functions and fragmentation functions. The laters describe the hadronization mechanisms of HQs into mesons and baryons. Therefore, mesons production fractions or baryon-to-meson rations make for excellent observables to study in order to understand 
these hadronization mechanisms.

By measuring $B_{s}^{0} / B^{+}$or $B_{s}^{0} / B^{0}$ ratios [6] in $p p$ collisions at $\sqrt{s}=7,8$ and $13 \mathrm{TeV}$, LHCb has studied the dependence of fragmentation functions $f_{s} / f_{u}$ and $f_{s} / f_{d}$ on $p_{\mathrm{T}}$ and rapidity for multiple colliding energies. While no rapidity dependence has been observed for the three datasets, a $p_{\mathrm{T}}$ dependent behaviour has been measured with 6 sigma significance, driven especially by the $13 \mathrm{TeV}$ sample (Fig. 1, right). Moreover, a $4.8 \sigma$ significance is seen for $f_{s} / f_{u}$ dependence on collision energy (Fig. 1, left), while no energy dependence is observed in the charm sector [9]. Likewise, the baryon-to-meson ratio $\Lambda_{b}^{0} /\left(\bar{B}_{0}+B^{-}\right)$has been measured to access the $f_{\Lambda_{b}^{0}} /\left(f_{u}+f_{d}\right)$ fragmentation fraction ratios in $p p$ at $\sqrt{s}=13 \mathrm{TeV}$ [7]. This new measurement is in a good agreement with previous results obtained at $\sqrt{s}=7 \mathrm{TeV}$ [8] for the $p_{\mathrm{T}}$ dependence of the $f_{\Lambda_{b}^{0}} /\left(f_{u}+f_{d}\right)$ ratio, with no rapidity dependence.

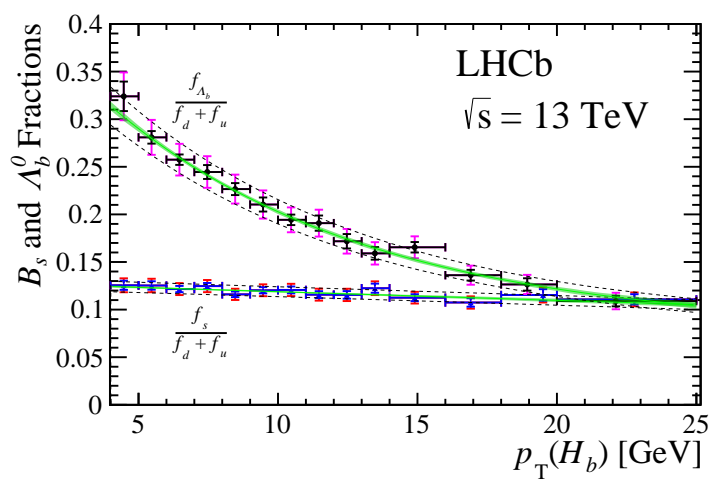

Figure 2: The $f_{s} /\left(f_{u}+f_{d}\right)\left(\overline{B_{s}^{0}} /\left(\bar{B}_{0}+B^{-}\right)\right)$and $f_{\Lambda_{b}^{0}} /\left(f_{u}+f_{d}\right)\left(\Lambda_{b}^{0} /\left(\bar{B}_{0}+B^{-}\right)\right)$yields ratios in bins of $p_{\mathrm{T}}(H b)$. The smaller (black, blue) error bars show the combined bin-by-bin statistical and systematic uncertainties, and the larger (ref, magenta) ones show the global systematics added in quadrature. The fits to the data are shown to guide the eye, with a $\pm 1 \sigma$ uncertainty band (green) on the fit shapes, and the dashed lines give the total uncertainty on the fit results.

\section{Recent results on $p \mathbf{P b}$ collisions: prompt double charm production}

The LHCb collaboration has recently measured the double prompt charm production at $\sqrt{s_{\mathrm{NN}}}$ $=8.16 \mathrm{TeV}$ [11]. The measurement is sensitive to the underlying event associated to a charm quark production, but could also give access to correlated parton distributions such as double transverse momentum dependent PDFs [10]. Experimentally, one can measure the associated production cross-section of two particle $(A$ and $B)$ in the same event $\left(\sigma^{A, B}\right)$ as a proxy for double parton scattering (DPS), and build an effective cross-section $\sigma_{e f f} \propto \frac{\sigma^{A} \sigma^{B}}{\sigma^{A B}}$, where $\sigma^{A, B}$ is the production cross-section of particle $A, B$, a proxy for single parton scattering (SPS). This $\sigma_{e f f}$ cross-section can be measured both in $p p\left(\sigma_{e f f, p p}\right)$ but also in pA collisions $\left(\sigma_{e f f, p A}\right)$. Simple scaling rules relates the two quantities [13], and any deviation of the measured $\sigma_{e f f, p A}$ compared to the predicted value from scaled $\sigma_{e f f, p p}$ would indicate additional correlation effects.

Fig. 3 shows the measured $\sigma_{e f f, p \mathrm{~Pb}}$ as a function of rapidity, for associated like-sign $D^{0}-D^{0}$ and 


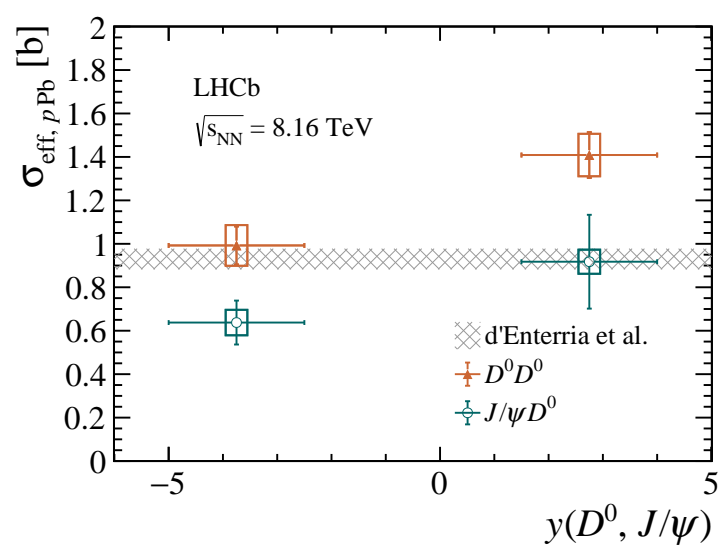

Figure 3: $\sigma_{e f f, p \mathrm{~Pb}}$ (in barn) as function of rapidity in the centre-of-mass system in $p \mathrm{~Pb}(y>0)$ and $\mathrm{Pbp}$ $(y<0)$ collisions at $\sqrt{s_{\mathrm{NN}}}=8.16 \mathrm{TeV}$ for like-sign $D^{0}-D^{0}$ (green) and $D^{0}-J / \psi$ (red) pairs. The error bars represent the statistical uncertainties, while boxes around the points represent the systematic uncertainties. The grey band is the theory prediction based on scaled $\sigma_{e f f, p p}$ results [13].

$D^{0}-J / \psi$ which are dominated by DPS production. The mean value from the theory $\sigma_{e f f, p \mathrm{~Pb}} \sim 0.9 \mathrm{~b}$ is consistent with the measured data, which indicates DPS/SPS enhancement by a factor three compared to $p p$ collisions. In addition, we observe that $\sigma_{e f f, p \mathrm{~Pb}}\left(D^{0}-D^{0}\right)>\sigma_{\text {eff, } p \mathrm{~Pb}}\left(D^{0}-J / \psi\right)$, but also $\sigma_{e f f, p \mathrm{~Pb}}\left(D^{0}-D^{0}, D^{0}-J / \psi\right)>\sigma_{e f f, P b p}\left(D^{0}-D^{0}, D^{0}-J / \psi\right)$. The two observations indicate DPS/SPS enhancement in $\left(D^{0}-D^{0}\right)$ compared to $D^{0}-J / \psi$ production, but also an enhancement in $p \mathrm{~Pb}$ with respect to $\mathrm{Pbp}$ collisions. However, one must be cautious about potential remaining SPS contamination in like-sign $D^{0}-D^{0}$ and $D^{0}-J / \psi$.

\section{Conclusions}

The large catalogue of datasets together with great performances make $\mathrm{LHCb}$ an ideal detector to study heavy-flavour productions. The forward acceptance of the detector and the reconstruction of tracks down to zero $p_{\text {T }}$ give access to a phase space coverage unique at the LHC. Recent results in $p p$ collisions allow to study HF hadronization mechanisms with great precision while new analyses are performed for the first time in high-energy $p \mathrm{~Pb}$ collisions such as the double charm production. With future upgrades and new data samples, the LHCb collaboration will continue to be a major actor in heavy-flavour measurements in small and large systems. 


\section{References}

[1] Alves Jr., A. A. and others, , JINST, Vol. 3, 2008

[2] The LHCb Collaboration, IJMP A, Vol. 30, No. 07, 1530022 (2015)

[3] The LHCb Collaboration, Phys. Rev. D99 052006 (2019)

[4] The LHCb Collaboration, Chin. Phys. C44 (2020) 022001

[5] The LHCb Collaboration, Phys. Rev. D99 (2019) 052011

[6] The LHCb Collaboration, Phys. Rev. Lett. 124, 122002 (2020)

[7] The LHCb Collaboration, Phys. Rev. D100 (2019) 031102

[8] The LHCb Collaboration, Phys. Rev. D85 (2012) 032008

[9] The LHCb Collaboration, Phys. Rev. D100 (2019) 112006

[10] The LHCb Collaboration, JHEP 1203 (2012) 089

[11] The LHCb Collaboration, LHCb-PAPER-2020-010

[12] M. G. Ryskin and A. M. Snigirev, Phys. Rev. D83 (2011) 114047

[13] D. d'Enterria, A. Snigirev, arXiv:1708.07519 